\title{
Serum Troponin I in Patients Suffering from Chronic Renal Disease
}

\section{with Acute Coronary Syndrome}

\section{Arash Forouzan ${ }^{1}$, Ali Delirrooyfard ${ }^{1 *}$, Akram Rezaei Panah ${ }^{1}$, Elham Maraghi}

'Department of Emergency Medicine, Ahvaz Jundishapur University of Medical Sciences, Ahvaz, Iran

${ }^{2}$ Department of Biostatistics and Epidemiology, Faculty of Public Health, Ahvaz Jundishapur University of Medical Sciences, Ahvaz, Iran

Study Area: Ahvaz, Iran

Coordinates: $31^{\circ} 19^{\prime} 13^{\prime \prime} \mathrm{N}$; $48^{\circ} 40^{\prime} 09^{\prime \prime E}$

Keywords:

Ethical standards have been met with informed consent. Ethical code :(IR.AJUMS.REC.1396.200)

\section{Introduction:}

Chronic Kidney Disease (CKD) is a fairly new term that has become popular after End-Stage Renal Disease (ESRD) was discovered. ESRD is the final stage of CKD and constitutes a clinical state associated with a uremic syndrome that has a very poor prognosis for the patient. CKD is the most important risk factor for ESRD (Levey \& Coresh, 2012). After its various aspects were identified, CKD became recognized as an actual disorder that entails significant complications besides ESRD. This disease is now known as a risk factor for conditions such as cardiovascular diseases, anemia, electrolyte disorders, bone diseases, cognitive and psychiatric disorders and many other diseases (Alan, 2015). Increased blood pressure, hyperlipidemia, hyperthyroidism, calcium and phosphorus metabolism disorder, aging, diabetes and other systemic diseases that affect cardiac function are profuse in CKD patients. Mortality occurs in these patients as a result of an increased frequency of atherosclerosis, myocardial infarction, left ventricular hypertrophy, congestive heart failure, hypertension, pericardial effusion and high tamponed (Lopez-Gomez et al., 1998). A large number of CKD patients are hospitalized

\section{Abstract}

Myocardial infarction is one of the common causes of death in patients with Chronic Kidney Disease (CKD). Cardiac troponins are myof ibril proteins. The present study aims to assess the serum troponin I level in CKD patients with acute coronary syndrome through a cross-sectional study conducted on stage- 3 to stage- 5 CKD patients. The patients' serum troponin I and biochemical factors were measured. Angiography was also performed and the results were compared with the serum levels of troponin I and biochemical factors. Out of 98 patients who took part in the present study, $19.4 \%$ had no venous obstruction, $30.6 \%$ with single-vein obstruction, $28.6 \%$ with double-vein obstruction and $21.4 \%$ with triple-vein obstruction. The angiography results were not significantly differed from BMI, the Glomerular Filtration Rate (GFR), serum creatinine and serum troponin I. No significant relationships were observed between age, creatinine, GFR, and troponin I in patients with no venous obstruction. A significant and positive relationship was observed between creatinine and troponin I in patients with single-vein obstruction. GFR was found to have a significant and negative relationship with creatinine and troponin I in patients with double- and triple-vein obstructions.

every year for the acute coronary syndrome (ACS), and a percentage of them have Acute Myocardial Infarction (AMI) and the rest suffer from unstable angina. AMI is known as a clinical disease caused by atherosclerosis of the arteries feeding the heart. The most common complaint in AMI patients is chest pain, which usually lasts for more than 15 minutes. AMI can also present with symptoms other than chest pain, which are known as angina equivalents and include sudden dyspnea, weakness and lethargy (Wright et al., 2002). For several years, especially in recent decades, new up-to-date diagnostic tools have become available to physicians for the diagnosis of AMI, and these early diagnoses have reduced the mortality rate associated with this condition and its potential complications. The World Health Organization criteria for the diagnosis of AMI include at least two of the three clinical symptoms indicating ischemic heart disease, including ECG variations and an increase and decrease in serum cardiac markers (Salehi Omran et al., 2005). Cardiac biomarkers are a useful tool for the diagnosis of AMI. Cardiac troponins are myof ibril proteins that include troponin $\mathrm{T}$, I and $\mathrm{C}$. The troponin complex regulates the process of skeletal muscle

*Corresponding Author: adelir2891@gmail.com 
contraction that occurs through calcium. Troponin $\mathrm{C}$ binds to calcium, troponin I binds to actin and the interaction of actin and myosin is thus inhibited, and troponin $\mathrm{T}$ binds to tropomyosin and makes the troponin complex connect to the thin filament (Farah \& Reinach, 1995). Troponin T and I are found in both the cardiac and skeletal muscles but are encoded with different genes and amino acid sequences, and antibodies specific to cardiac troponin $\mathrm{T}$ and $\mathrm{I}$ are thus synthesized (Antman et al., 1996). The cardiac troponin I test is specific to the myocardial tissue, and its values above the reference are regarded as an indicator of AMI in people with normal kidney function (Adams et al., 1993). Troponin I values may be higher in CKD patients than in others due to minor myocardial damage, which is not detectable by regular cardiac imaging (Martin et al., 1998). A number of studies have demonstrated the value of troponin I in the diagnosis of acute coronary syndrome in CKD patients, but others have emphasized the inability of this test in diagnosing acute coronary syndrome (Jafari Fesharaki et al., 2016). The present study was conducted to investigate serum troponin I levels in CKD patients with acute coronary syndrome.

\section{Methodology:}

This study was conducted following to the Strengthening the Reporting of Observational Studies in Epidemiology (STROBE) (von Elm et al., 2014). The descriptive epidemiologic study was conducted on patients presenting to the emergency departments of Imam Khomeini and Golestan hospitals in Ahwaz in 2017-18.

Participants were then Stage- 3 to stage -5 CKD patients with chest pain referred for angiography were included in the study after submitting their written consent. The exclusion criteria consisted of being a critical patient, the loss of consciousness, having no kidney problems, having kidney problems but no chest pain and unwillingness to undergo angiography despite being its candidate.

Data collection: stages 3 to 5 of CKD were defined by a $\mathrm{GFR}<6 \mathrm{omL} / \mathrm{min} / 1.73 \mathrm{~m} 2$ of the body surface for over three months. A retrosternal pain initiated acutely and abated by sublingual nitroglycerine and often lasting over 20 minutes was also taken as chest pain. The results of the physical examination and cardiovascular nuclear scan (if performed) and the demographic details, including age, gender, weight, height and smoking status, were recorded. On the day of the visit, the patients' weight was carefully measured with minimal clothing without shoes using a scale, and their height was measured in a standing position with no shoes using a wall-mounted stadiometer with a 1$\mathrm{cm}$ precision and with the buttocks, shoulders and heels touching the wall and the head looking forward. BMI was calculated as the weight to height ratio $\left(\mathrm{kg} / \mathrm{m}^{2}\right)$. Serum lipids, creatinine, and troponin I levels was measured using standard kits and relevant laboratory techniques in the central laboratory. The patients underwent angiography by a specialist. Cardiac biomarkers and other variables were ultimately assessed in the patients and compared to the angiography results.

Statistical analysis: the collected data were analyzed in SPSS-22. The quantitative variables were reported as mean with standard deviation and the qualitative variables as frequency (percentage). The normality of the quantitative variables was assessed using the Shapiro-Wilks test. The relationships were assessed using the Pearson or Spearman correlation tests for the quantitative variables and using the Chi-square test for the qualitative variables. The quantitative variables were compared between the two groups using the independent t-test or its non-parametric equivalent (Mann-Whitney's test). The quantitative variables were compared between more than two groups using the ANOVA or its equivalent (the Kruskal-Wallis test). The tests were two-tailed and the level of statistical significance was set at $\mathrm{p}<0.05$.

\section{Results:}

A total of 98 patients participated in this study. Their mean age was $59.82 \pm 11.4$, and $48(49 \%)$ were male and $50(51 \%)$ were female. According to the angiographic results,19 patients $(19.4 \%)$ did not have venous obstruction and 79 (80.6\%) did have venous obstruction, including 30 (30.6\%) with single-vein obstruction, 28 (28.6\%) with double-vein obstruction and $21(21.4 \%)$ with triple-vein obstruction (Table-1). No significant difference was observed in the frequency of angiographic results in the study population ( $p>0.997)$. No significant differences were observed in the angiographic results by gender, smoking status, blood pressure or serum lipids. Serum creatinine and serum troponin I were significantly higher in the patients with venous obstruction compared to those without. The mean BMI and GFR were significantly lower in patients with obstruction compared to those without $(\mathrm{p}<0.05)$. The results showed no significant relationship between the noted factors in the patients without venous obstruction (Table-1).

In patients without venous obstruction, no significant relationships were observed between creatinine and troponin I ( $>0.095, r=0.200)$ or between GFR and creatinine $(\mathrm{p}>0.768, \mathrm{r}=-0.209)$ and GFR and troponin I ( $\mathrm{p}>0.101, \mathrm{r}=-0.374)$; (Fig.-1).

A positive and significant relationship was observed between creatinine and troponin $\mathrm{I}$ in the patients with single-vein obstruction $(\mathrm{p}<0.015, \mathrm{r}=0.254)$. There was no significant negative relationship between GFR and creatinine ( $\mathrm{p}>0.335, \mathrm{r}=-0.015)$ or between GFR and troponin I ( $\mathrm{p}>0.066, \mathrm{r}=-0.015)$; (Fig.-2).

In addition, a positive and signif icant relationship was observed between troponin I and creatinine $(\mathrm{p}<0.00$, $\mathrm{r}=\mathbf{0 . 2 5 4}$ ) and significant negative relationships were 
observed between GFR and creatinine ( $\mathrm{p}<0.003, \mathrm{r}=-0.416)$ and between GFR and troponin I ( $\mathrm{p}<0.00, \mathrm{r}=-0.588)$ in the patients with double-vein obstruction (Fig.-3). In the patients with triple-vein obstruction, significant negative relationships were observed between GFR and creatinine $(\mathrm{p}<0.006, \mathrm{r}=-0.466)$ and between GFR and troponin I $(\mathrm{p}<0.016, \mathrm{r}=-0.581)$. A positive and significant relationship was also observed between troponin I and creatinine ( $\mathrm{p}<0.001, \mathrm{r}=0.509$ ); (Fig. -4 ).

Table-1: Baseline information of patients as pervessel obstruction

\begin{tabular}{|c|c|c|c|c|c|}
\hline \multicolumn{6}{|c|}{ Demographics } \\
\hline $\begin{array}{l}\text { Total } \\
(\mathrm{n}=98)\end{array}$ & $\begin{array}{l}\text { Lack of } \\
\text { obs. }(n=19)\end{array}$ & $\begin{array}{l}1 \text { vessel } \\
\text { obs. }(n=30)\end{array}$ & $\begin{array}{l}2 \text { vessel } \\
\text { obs. }(n=28)\end{array}$ & $\begin{array}{l}3 \text { vessels } \\
\text { obs. }(n=21)\end{array}$ & $\begin{array}{l}\text { 'P'- } \\
\text { value }\end{array}$ \\
\hline \multicolumn{6}{|c|}{ Age (Years) } \\
\hline $\begin{array}{l}59.82 \pm 11.4 \\
\text { Male (\%) }\end{array}$ & $56.26 \pm 13.1$ & $59 \pm$ & 62.8 & 60. & $>0.085$ \\
\hline \multicolumn{5}{|c|}{ BMI $\left(\mathrm{Kg} / \mathbf{m}^{2}\right)$} & $>0.997$ \\
\hline $\begin{array}{l}30.77 \pm 4.53 \\
\text { Clinical Ch } \\
\text { Current sr }\end{array}$ & $\begin{array}{l}34.12 \pm 3.16 \\
\text { aracteristics } \\
\text { noker }(\%)\end{array}$ & .18 & 4.80 & .22 & $<0.000$ \\
\hline $\begin{array}{l}43(44.9) \\
\text { Hypertens }\end{array}$ & $\begin{array}{r}7(16.3) \\
\text { ion }(\%)\end{array}$ & $8(1 \xi$ & 16( & 12( & $>0.058$ \\
\hline $\begin{array}{l}68 \text { (69.4) } \\
\text { Laboratory }\end{array}$ & $9(13.2)$ & $24(35 \cdot 3)$ & $20(29.4)$ & 15( & $>0.11$ \\
\hline High bloo & d lipids(\%) & & & & \\
\hline $\begin{array}{l}54(55.1) \\
\text { Serum cre }\end{array}$ & $\begin{array}{l}13(24.1) \\
\text { atinine }(\mathrm{mg}\end{array}$ & $\begin{array}{l}20(37) \\
/ \text { dl) }\end{array}$ & $12(22.2)$ & $9(16.7)$ & $>0.114$ \\
\hline $\begin{array}{l}2.15 \pm 0.60 \\
\text { Serum Glo } \\
46.71 \pm 10.55 \\
\text { Troponin }\end{array}$ & $\begin{array}{l}1.79 \pm 0.32 \\
\text { merular Fil } \\
53.09 \pm 6.37 \\
\text { I (ng/dl) }\end{array}$ & $\begin{array}{c}1.91 \pm 0.36 \\
\text { tration Rat } \\
50.59 \pm 7.72\end{array}$ & $\begin{array}{l}2.31 \pm 0.65 \\
\text { te (GFR) (m } \\
44.5^{2 \pm 11.02}\end{array}$ & $\begin{array}{c}2.61 \pm 0.85 \\
1 \mathrm{~L} / \mathrm{min} / 1.73 \\
38.30 \pm 10.52\end{array}$ & $\begin{array}{l}<0.000 \\
\left.\mathrm{~m}^{2}\right) \\
<0.000\end{array}$ \\
\hline $1.47 \pm 2.55$ & $0.094 \pm 0.41$ & $0.30 \pm 1.07$ & $1.72 \pm 2.70$ & $4.5^{2} \pm 3.00$ & $<0.000$ \\
\hline
\end{tabular}

Discussion:

According to the results obtained, the mean troponin I level was $0.094 \pm 0.41 \mathrm{ng} / \mathrm{dL}$ in those with no vein obstruction, $0.03 \pm 1.07 n g / d L$ in single-vein obstruction, $1.72 \pm 2.70 \mathrm{ng} / \mathrm{dL}$ in double-vein obstruction and $4.52 \pm 3.00 \mathrm{ng} / \mathrm{dL}$ in triplevein obstruction, comprising a significant difference. In line with the present findings, Flores et al. (2006) reported signif icantly higher troponin I level in patients with CKD compared to those without. In a study conducted by Giocoechea et al. (2005), CKD patients with cardiovascular diseases had high troponin I levels compared to others. In a study by Chen et al. (2013), higher levels of troponin I was observed in $43.34 \%$ of the CKD patients with acute coronary syndrome and $26.03 \%$ of the CKD patients without the acute coronary syndrome, which suggests higher troponin I levels in patients with the non-acute coronary syndrome. Apple et al., (1997) reported on the 16 CKD patients undergoing hemodialysis had a history of ischemic heart disease (unstable angina), and their troponin I level was higher than the reference $(0.8 \mathrm{ng} / \mathrm{ml})$. In a study conducted by Fesharaki et al. (2016), the mean troponin I level was $0.95 \pm 1.44 \mathrm{ng} / \mathrm{dL}$ in the patients with normal angiography
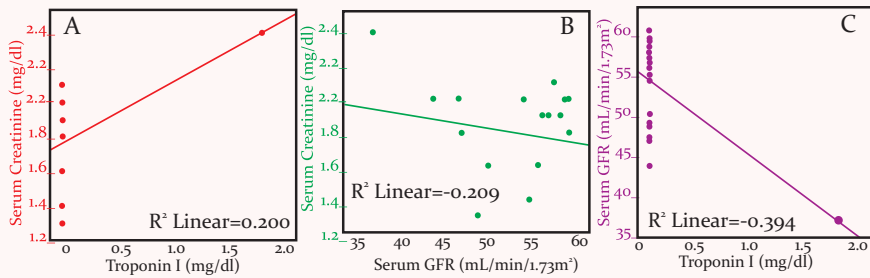

Figure-1: Plot between creatinine \& troponin I (A). Correlation between GFR \& creatinine (B).Correlation between GFR \& troponin I (C).
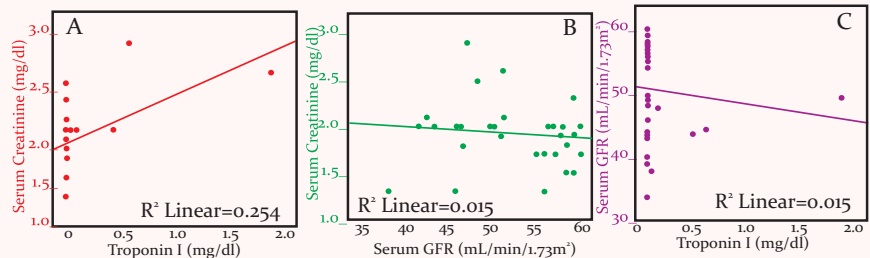

Figure-2: Plot between creatinine \& troponin I in the patients with single-vein (A). Correlation between GFR \& creatinine (B).Correlation between GFR \& troponin I (C).
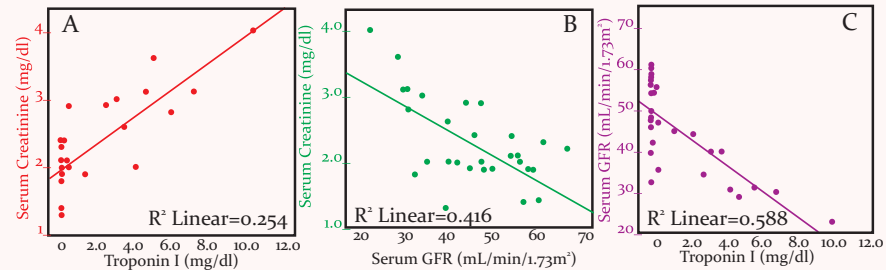

Figure-3: Plot between creatinine \& troponin I in the patients with double-vein obstruction (A). Correlation between GFR \& creatinine (B). Correlation between GFR \& troponin I (C).
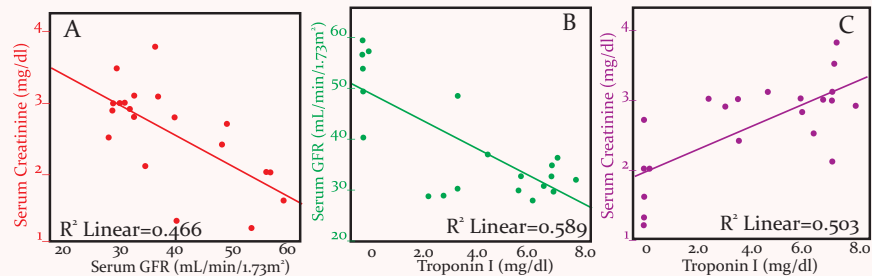

Figure-4: Plot correlation between GFR \& creatinine and troponin I in the patients with triple-vein (A). Correlation between GFR \& troponin I (B).Correlation between creatinine \& troponin I (C).

and $1.82 \pm 2.96 \mathrm{ng} / \mathrm{dL}$ in those with abnormal angiography. However, we didn't get any significant differences in terms of gender, smoking status, blood pressure or serum lipids in the angiographic results. Moreover, the mean BMI, GFR, and serum creatinine was higher in patients with venous obstruction. A number of studies have reported BMI, blood pressure, hyperlipidemia and smoking as risk factors for acute coronary syndrome in CKD patients, although some other studies did not report a signif icant difference. Ohtake et al. (2005), reported that the coronary artery stenosis in stage-5 renal failure or ESRD patients was related signif icantly to the total triglyceride, total cholesterol, and LDL. Further, as per Hase et al. (1993), the risk factors of acute coronary syndrome in 101 CKD patients, including some who were undergoing hemodialysis existed. And the 
step-wise logistic regression analysis of the risk factors showed that the prevalence of acute coronary syndrome had a significant relationship with diabetes and smoking. In another study of Jungers et al. (1977), atherosclerotic coronary artery disease was found to have a significant relationship with smoking and high systolic blood pressure. Moreover, CKD patients with atherosclerotic coronary artery disease had lower levels of HDL and higher levels of triglyceride, LDL, and homocysteine. Muntner et al. (2005) reported that smoking, high blood pressure, high blood fat, diabetes, high BMI and high waist circumference were risk factors related to the prevalence of coronary heart disease in CKD patients. The researchers argued that the risk factors of coronary heart disease in CKD patients are the same as those in the general public, but given the higher prevalence of heart disease in CKD patients, these risk factors have to be controlled. In the present study, venous obstruction was statistically higher in those with a higher BMI and also higher in those with hyperlipidemia and high BP and also in the smokers, although not supported by Statistics.

Smoking disrupts the function of the coronary capillaries and causes myocardial damage by inducing oxidative stress. High blood pressure is also associated with an increased risk of coronary artery disease and left ventricular hypertrophy in patients with CKD. The increase in blood pressure is caused by an increased volume of fluids, the activation of the renin-angiotensin system and increased nitric oxide inhibitors in the blood circulation, such as ADMA. Left ventricular hypertrophy causes an increase in left ventricular end-diastolic pressure and a reduction in subendocardial coronary blood flow, which then leads to the necrosis of small myocardial cells, apoptosis, the leakage of intracellular content due to the enhanced permeability of myocytes, increased levels of troponin $\mathrm{T}$ and I and ultimately an increased risk of ischemic heart disease (Foley et al., 1995). Hyperlipidemia is one of the known risk factors for coronary artery disease and atherosclerosis progress, and the increase in certain types of plasma lipids has been shown to exacerbate the atherosclerotic engagement of the coronary arteries. Recent evidence suggests that a slight increase in triglycerides leads to an increased risk of coronary incidents and hastens coronary artery disease and also leads to the formation of new lesions and further extends coronary artery engagement. The relationship between serum blood lipids and obstructive coronary artery disease is well known, although only a few studies have been conducted on the role of lipoproteins as indices of the severity of coronary artery engagement (Korhonen et al., 1996).

Our study showed that the troponin I level has no signif icant relationships with creatinine or GFR in patients with no venous obstruction in the angiographic results, but in patients with venous obstruction, it has a positive and signif icant relationship with creatinine and a negative and significant relationship with GFR. In line with the present findings, Fesharaki et al. (2016) showed that serum creatinine was significantly higher in patients with troponin I $>0.1 \mathrm{ng} / \mathrm{ml}$ than in those with troponin $\mathrm{I}<0.1 n g / \mathrm{ml}$. In addition, GFR was significantly lower in patients with troponin I>0.1ng/ml (Fesharaki et al., 2016). In a study of Zand Parsa et al. (2012), troponin I had no significant relationships with GFR or serum creatinine in CKD patients with no heart problems, which agrees with the present findings since no relationships were observed between troponin I and GFR and creatinine in the patients with no venous obstruction. Otsuka et al. (2010) reported that troponin $\mathrm{T}$ had a signif icant relationship with the risk factors of heart disease, including age, blood flux, GFR, smoking and left ventricular hypertrophy. Troponin T had an inverse relationship with GFR. In fact, troponin I and T showed an increase in CKD patients with no cardiac disease, which is probably due to the reduced troponin I clearance.

\section{Conclusion:}

The present findings showed no significant differences in the severity of acute coronary syndrome by gender, smoking status, blood pressure, and serum lipids, but signif icant differences were observed in this severity by the mean BMI, GFR, serum creatinine and serum troponin I. Moreover, in patients with no venous obstruction, no significant relationships were found between age, serum creatinine, GFR or troponin I. A positive and significant relationship was observed between creatinine and troponin I in the patients with single-vein obstruction and a negative and significant relationship between GFR and creatinine and between GFR and troponin I and a positive and significant relationship between troponin I and creatinine in the patients with double-vein and triple-vein obstruction.

\section{Acknowledgment:}

The authors wish to acknowledge the support of the deputy of research affairs of the Ahvaz Jundishapur University of Medical Sciences as part of Akram Rezaei Panah MD thesis under the research code $\mathrm{GP} 95177$.

\section{References:}

Adams, J.E., Bodor, G.S., Davila-Roman, V.G., Delmez, J.A., Apple, F.S., Ladenson, J.H. \& Jaffe, A.S. (1993): Cardiac troponin I. A marker with high specificity for cardiac injury. Circulation, 88(1):101-106.

Alan, S. (2015): Claudins and the kidney. J. Am. Soc. Nephrol., 26(1):11-19.

Antman, E.M., Tanasijevic, M.J., Thompson, B., Schactman, M., McCabe, C.H., Cannon, C.P., Fischer, G.A., Fung, A.Y., Thompson, C., Wybenga, D. \& Braunwald, E. (1996): Cardiacspecific troponin I levels to predict the risk of mortality in patients with acute coronary syndromes. New Eng. J. Med., 335(18):1342-1349.

Apple, F.S., Sharkey, S.W., Hoeft, P., Skeate, R., Voss, E., Dahlmeier, B.A. \& Preese, L.M. (1997): Prognostic value of serum cardiac 


\section{ORIGINAL ARTICLE}

troponin I and T in chronic dialysis patients: a 1-year outcomes analysis. Am. J. Kidney Dis., 29(3):399-403.

Chen, S., Huang, C., Wu, B., Lian, X., Mei, X. \& Wan, J. (2013): Cardiac troponin I in non- acute coronary syndrome patients with chronic kidney disease. PLoS One, 8(12):e82752. PLoS One. 2013; 8(12): 882752

Farah, C.S. \& Reinach, F.C. (1995): The troponin complex and regulation of muscle contraction. FASEB J., 9(9):755767.

Flores, L.M., Hernandez Dominguez, J.L., Otero, A. \& Gonzalez Juanatey, J.R. (2006): Cardiac troponin I determination in patients with chronic renal failure. Nefrologia, 26(1):107-112.

Foley, R.N., Parfrey, P.S., Harnett, J.D., Kent, G.M., Martin, C.J., Murray, D.C. \& Barre, P.E. (1995): Clinical and echocardiographic disease in patients starting end-stage renal disease therapy. Kidney Int., 47(1):186-192.

Goicoechea, M., de Vinuesa, S.G., Gomez-Campdera, F. \& Luno, J. (2005): Predictive cardiovascular risk factors in patients with chronic kidney disease (CKD). Kidney Int.Suppl., 67(93):S3538.

Hase, H., Nakamura, R., Ui, K., Imamura, Y., Inishi, Y., Jyoki, N., Yasuda, C., Ishikawa, H., Yabuki, S. \& Yamaguchi, T. (1993): Risk factors for coronary artery disease in Japanese patients with chronic renal failure. Nihon Jinzo Gakkai shi, 35(6):751756.

Jafari Fesharaki, M., Alipour Parsa, S., Nafar, M., Ghaffari-Rahbar, M., Omidi, F. \& Karimi-Sari, H. (2016): Serum Troponin I Level for Diagnosis of Acute Coronary Syndrome in Patients with Chronic Kidney Disease. Iran. J. Kidney Dis., 10(1):11-16.

Jungers, P., Massy, Z.A., Nguyen Khoa, T., Fumeron, C., Labrunie, M., Lacour, B., Descamps-Latscha, B. \& Man, N.K. (1997): Incidence and risk factors of atherosclerotic cardiovascular accidents in predialysis chronic renal failure patients: a prospective study. Nephrol. Dial. Transplant., 12(12):25972602.

Korhonen, T., Savolainen, M.J., Koistinen, M.J., Ikaheimo, M., Linnaluoto, M.K., Kervinen, K. \& Kesaniemi, Y.A. (1996): Association of lipoprotein cholesterol and triglycerides with the severity of coronary artery disease in men and women. Atherosclerosis, 127(2):213-220.

Levey, A.S. \& Coresh, J. (2012): Chronic kidney disease. Lancet, 379(9811):165-180.
Ambient Science, 2019: Vol. 06h(2); 77-81 DOI:10.21276/ambi.2019.06h.2.oa18

Lopez-Gomez, J.M., Verde, E. \& Perez-Garcia, R. (1998): Blood pressure, left ventricular hypertrophy and long-term prognosis in hemodialysis patients. Kidney Int. Supp., 68:S9298.

Martin, G.S., Becker, B.N. \& Schulman, G. (1998): Cardiac troponin-I accurately predicts myocardial injury in renal failure. Nephrol Dial Transpl.. 13(7):1709-1712.

Muntner, P., He, J., Astor, B.C., Folsom, A.R. \& Coresh, J. (2005): Traditional and nontraditional risk factors predict coronary heart disease in chronic kidney disease: results from the atherosclerosis risk in communities study.J.Am. Soc. Nephrol. (JASN), 16(2):529-538.

Ohtake, T., Kobayashi, S., Moriya, H., Negishi, K., Okamoto, K., Maesato, K. \& Saito, S. (2005): High prevalence of occult coronary artery stenosis in patients with chronic kidney disease at the initiation of renal replacement therapy: an angiographic examination. L. Am. Soc. Nephrol. (JASN), 16(4):1141-1148.

Otsuka, T., Kawada, T., Ibuki, C. \& Seino, Y. (2010): Association between high-sensitivity cardiac troponin $\mathrm{T}$ levels and the predicted cardiovascular risk in middle-aged men without overt cardiovascular disease. Am. Heart I., 159(6):972978.

Salehi Omran, M.T., Khososi Niaki, M.R., Hajian, K.D., Soleimani, M., Ghasemzadeh, S.M. \& Saberian, F. (2004): Measurement of cardiac troponin I and troponin $\mathrm{T}$ in patients with acute myocardial infarction. Horizon of Med. Sci., 10(3):49-52.

von Elm, E., Altman, D.G., Egger, M., Pocock, S.J., Gotzsche, P.C. \& Vandenbroucke, J.P. Initiative S. (2008): The Strengthening the Reporting of Observational Studies in Epidemiology (STROBE) Statement: guidelines for reporting observational studies. Int. J. Surg. (London, England), 12(12):1495-1499.

Wright, R.S., Reeder, G.S., Herzog, C.A., Albright, R.C., Williams, B.A., Dvorak, D.L., Miller, W.L., Murphy, J.G., Kopecky, S.L. \& Jaffe, A.S. (2002): Acute myocardial infarction and renal dysfunction: a high-risk combination. Ann. Int. Med., 137(7):563-570.

Zand Parsa, A.F., Abdolahi, A. \& Mahdavimazdeh, M. (2012): Is cardiac biomarkers and left ventricular function affected by chronic kidney disease? Indian Heart I., 64(5):479-483. 